\title{
Mismatch Negativity Indices as a Prognostic Factor for Remission in Schizophrenia
}

\author{
Ji Sun Kim', Young Joon Kwon', Hwa Young Lee', Ho-Sung Lee', Sungkean Kim³, Se-hoon Shim ${ }^{1}$ \\ ${ }^{1}$ Departments of Psychiatry, ${ }^{2}$ Pulmonology and Allergy, Soonchunhyang University Cheonan Hospital, Cheonan, ${ }^{3}$ Department of Biomedical \\ Engineering, Hanyang University, Seoul, Korea
}

\begin{abstract}
Objective: Mismatch negativity $(\mathrm{MMN})$ is known to be associated with neuro-cognition and functional outcomes. Remission and recovery rates are related to the neuro-cognition of patients with schizophrenia. The present study explored the relationship of MMN with remission in patients with schizophrenia.

Methods: Forty patients with schizophrenia were recruited and divided into two groups, with or without remission, according to the Remission in Schizophrenia Working Group criteria (RSWGcr). Symptom severity (Positive and Negative Syndrome Scale, PANSS), cognitive function, functional outcome, and MMN of the patients were evaluated. A regression analysis was used to identify the factors that significantly predicted symptom improvement and remission including $M M N$ at frontal site assessed at baseline, and anticipated clinical variables as predictive factors.

Results: MMN amplitudes in frontal sites were further decreased in the groups without remission compared to the groups with remission. MMN amplitude was significantly correlated with measures of symptom change and functional outcome measurements in patients with schizophrenia. Regression analysis revealed that symptom severity and MMN significantly predicted remission in patients with schizophrenia. Symptom improvement significantly predicted PANSS at baseline, illness duration, and antipsychotic dose, as did MMN amplitude at frontal site.

Conclusion: Our results suggest that MMN reflected symptom improvement and remission in patients with schizophrenia. MMN indices appear to be promising candidates as predictive factors for schizophrenia remission.
\end{abstract}

KEY WORDS: Schizophrenia; Remission; Symptom improvement; Mismatch negativity.

\section{INTRODUCTION}

Schizophrenia is a chronic psychiatric disorder characterized by both clinical symptoms, including delusions, hallucinations, anhedonia, and affective flattening, and cognitive impairments [1]. Remission is considered an important target for the treatment of schizophrenia $[2,3]$. Recent studies have revealed that symptomatic remission could be attributed to better functional prognosis and quality of life in patients with schizophrenia [4,5]. In this respect, predicting remission and finding factors associated with remission in schizophrenia patients has been

Received: June 10, 2019 / Revised: August 6, 2019

Accepted: September 2, 2019

Address for correspondence: Se-hoon Shim

Department of Psychiatry, Soonchunhyang University Cheonan

Hospital, 31 Suncheonhyang 6-gil, Dongnam-gu, Cheonan

31151, Korea

E-mail: shshim2k@daum.net

ORCID: https://orcid.org/0000-0002-3137-6591 of great interest to clinicians [6].

The remission rate is related to the neuro-cognition of patients with schizophrenia [7]. That is, the neuro-cognition of patients with schizophrenia is important to achieving remission. Interestingly, mismatch negativity $(\mathrm{MMN})$ is known to be associated with neuro-cognition and functional outcomes [8,9]. Näätänen et al. [10] comprehensively reviewed studies which closely correlated $M M N$ with cognitive status. MMN is an event-related potential component when a sequence of relatively standard stimuli is interrupted by the infrequent presentation of deviant stimuli [11] and it represents preattentive auditory processing [12].

Abnormally decreased MMN has often been reported in schizophrenia $[13,14]$. MMN deficits may index both ongoing disease processes associated with illness progression, as well as with premorbid neurocognitive impairment [15]. Additionally, impaired MMN in patients

(c) This is an Open-Access article distributed under the terms of the Creative Commons Attribution Non-Commercial License (http://creativecommons.org/licenses/by-nc/4.0) which permits unrestricted non-commercial use, distribution, and reproduction in any medium, provided the original work is properly cited. 
with schizophrenia and its association with impaired functional status have been consistently reported $[16,17]$. Regarding the association of MMN with neuro-cognition, functioning, and illness progression in schizophrenia, it could be speculated that MMN affects remission and recovery of schizophrenia.

However, no previous studies have completely explored the relationships between early auditory processing MMN and remission in patients with schizophrenia. A recent study reported that $M M N$ was a putative predictor of prognosis, regardless of the transition to psychotic disorders in subjects at high clinical risk [18]. It revealed that MMN could predict the remission of subjects at high clinical risk [18], but the population of the study had not yet been diagnosed with schizophrenia.

Meanwhile, despite the clinical importance of remission, assessment of remission is lacking. One effort to define remission was the publication of the Remission in Schizophrenia Working Group criteria (RSWGcr) [19]. The RSWG proposed a consensus definition of symptomatic remission in schizophrenia and developed specific operational criteria for its assessment [20]. It has proved to be conceptually viable and easy to use, both in clinical trials and clinical practice [21].

The present study explored the relationships of MMN with remission in patients with schizophrenia using the reliable RSWGcr. The study aimed to determine whether baseline MMN predicted remission six months later [22]. We hypothesized that patients not going into remission at follow-up would show smaller baseline MMN amplitudes than those in remission. We also hypothesized that the baseline MMN amplitude, as well as neuro-cognition and some clinical characteristics affecting remission, would predict later remission.

\section{METHODS}

\section{Participants}

Participants were recruited from the Psychiatry Department of Soonchunhyang University Cheonan Hospital, Korea. Patients with schizophrenia were diagnosed according to the Structured Clinical Interview for Diagnostic and Statistical Manual of Mental Disorders, 4th edition (DSM-IV) Axis I Psychiatric Disorders [23]. Only patients with medication adherence confirmed by their guardians were enrolled this study. The study was performed on 40 patients with schizophrenia (20 male and 20 female), with a mean age of $32.59 \pm 12.33$ years. Participants with any history of neurological or other severe medical diseases were excluded from the study through the initial screening interviews. None of the patients had mental retardation or alcohol abuse, electroconvulsive therapy, or head injury. Each participant had normal hearing ability, confirmed by the $512-\mathrm{Hz}$ tuning fork test [24], and all were identified as right-handed. All patients were taking oral atypical antipsychotics (risperidone, $\mathrm{n}=15$; olanzapine, $\mathrm{n}=12$; amisulpride, $\mathrm{n}=8$; aripiprazole, $\mathrm{n}=3$; or ziprasidone, $n=2$ ). None of the patients were taking injectable medications. This study was approved by the Institutional Review Board and Ethics Committee of Soonchunhyang University Cheonan Hospital and all experimental protocols were approved by the committee (2018-10-010-001). The study was performed in accordance with approved guidelines.

\section{Assessment}

Patients with schizophrenia were assessed using the Positive and Negative Syndrome Scale (PANSS), the Hamilton Depression Scale (HAM-D), and the Global Assessment of Functioning (GAF) at baseline. The intelligence quotient (IQ) of all participants was also measured with the abbreviated form of the Korean Wechsler Adult Intelligence Scale-Revised (K-WAIS-R) at baseline. Antipsychotic medication use at follow-up was assessed as the mean risperidone equivalent dose [25]. PANSS was re-administered to all participants six months later to evaluate remission [22]. GAF was also re-assessed to evaluate functional status at follow-up. Forty patients with schizophrenia were recruited and divided into two groups, with or without remission, according to the RSWGcr [19]. RSWGcr, based on ratings of eight focal symptoms on positive, negative, and general psychopathology subscales of the PANSS (P1, P2, P3, N1, N4, N6, G5, G9), were applied to determine clinical remission. Patients were judged to be in clinical remission according to severity criteria (scores obtained on each of these items had to be $\leq 3$ points, indicating mild severity of symptoms) [19]. In this study, clinical remission was evaluated, taking into account the severity criteria alone, excluding the duration criterion (remission maintained for six months) [26]. PANSS administrations were conducted by two psychiatrists (JSK \& SHS) and the interrater reliability value 
was 0.81 . In the study, 19 patients met remission criteria and 21 did not meet the criteria.

\section{Data Acquisition and Analysis}

During the electroencephalogram (EEG) task, each participant was tested in a sound-attenuated EEG room. EEG data were acquired using a NeuroScan SynAmps amplifier (Compumedics USA, El Paso, TX, USA) with 64 $\mathrm{Ag}-\mathrm{AgCl}$ electrodes mounted on a Quik Cap, using an extended 10-20 placement scheme. The ground electrode was located on the forehead and the physically-linked reference electrode was attached to both mastoids. The vertical electrooculogram (EOG) was positioned above and below the left eye and the horizontal EOG was recorded at the outer canthus of each eye. The impedance was kept below $5 \mathrm{k} \Omega$. All data were processed with a $0.1-100 \mathrm{~Hz}$ band-pass filter and sampled at 1,000 Hz. The recorded EEG data were preprocessed using CURRY 8. Gross artifacts were rejected through visual inspection by a trained person with no prior information regarding the origin of the data. Artifacts related to eye movement or eye blinks were removed using a mathematical procedure implemented in the preprocessing software. Especially, horizontal eye movements were rejected by visual inspection and vertical eye movements such as eye blinking were removed by method using covariance and regression for reducing ocular artifact [27]. The data were filtered using a $0.1-30 \mathrm{~Hz}$ band-pass filter and epoched from $100 \mathrm{milli}$ seconds pre-stimulus to 600 milliseconds post-stimulus. The epochs were subtracted from the average value of the pre-stimulus interval for baseline correction. If any remaining epochs contained significant physiological artifacts (amplitude exceeding $\pm 75 \mu \mathrm{V}$ ) in any of the 62 electrode sites (except M1 and M2), they were excluded from further analysis. Only artifact-free epochs were averaged across trials and participants for event related potential (ERP) analysis.

Stimulus presentation and data synchronization with the EEG were conducted with E-prime (Psychology Software Tools, Pittsburgh, PA, USA). The auditory stimuli consisted of sounds at $85 \mathrm{~dB}$ sound pressure level and 1,000 $\mathrm{Hz}$. The subjects were asked to concentrate on a "Where's Wally?" picture book without paying attention to sound. The MMN was extracted separately for each subject by subtracting the ERPs elicited by standard stimuli from those elicited by deviant stimuli. Deviant tones lasting
100 milliseconds were presented randomly, interspersed with standard tones lasting 50 milliseconds $(20 \%$ and $80 \%$ probabilities, respectively). Auditory stimulation included 400 stimuli with a 1,500 milliseconds inter-stimulus interval (10 milliseconds rise and 10 milliseconds fall). These stimuli were presented through MDR-D777 headphones (Sony, Tokyo, Japan). The peak amplitude and latency of the MMN were determined as the most negative peak between 130 milliseconds and 250 milliseconds of the subtracted waveform using a peak detection method at six electrode sites (Fz, F3, F4, FCz, FC3, and FC4) [18].

\section{Statistical Analysis}

Independent sample $t$ tests were used to compare the demographic and clinical characteristics between the with or without remission groups. A chi-squared analysis or Fisher's exact test was used for categorical data. In order to investigate group differences in MMN amplitude, repeated measures analysis of variance (ANOVA) with electrode site as within-group factor and each compared group (remission vs. non-remission) as between group factor was performed. Group $\times$ electrode interactions was decomposed using one-way ANOVA, with False Discovery Rate (FDR). Additionally, for post-hoc analysis, the FDR was also applied to perform multiple testing correction. In addition, the relationship of MMN peak amplitude to PANSS changes and re-measured GAF scores in patients with schizophrenia was analyzed by partial Pearson's correlation to control for age and sex as covariates. A binary logistic regression with a backward selection method was used to identify the factors predicting remission. A multiple regression analysis with a backward selection method was used to identify the factors that significantly predicted symptom improvement. The anticipated predictive factors included MMN peak amplitude at Fz assessed at baseline, age, age at onset, illness duration, PANSS score at baseline, and final dose of antipsychotic medication. The significance level was set at $p<0.05$ (two-tailed). Statistical analyses were performed using IBM SPSS ver. 21 (IBM Corp., Armonk, NY, USA).

\section{RESULTS}

\section{Participants}

There was no significant group difference according to 
Table 1. Comparison of baseline demographic and clinical symptom characteristics between participants with and without remission

\begin{tabular}{lccc}
\hline \multicolumn{1}{c}{ Variable } & With remission $(\mathrm{n}=19)$ & Without remission $(\mathrm{n}=21)$ & $p$ value \\
\hline Age $(\mathrm{yr})$ & $28.32 \pm 7.52$ & $28.86 \pm 7.85$ & 0.83 \\
Sex & & & \\
$\quad$ Male & $11(57.9)$ & $9(42.9)$ & 0.53 \\
$\quad$ Female & $8(42.1)$ & $12(57.1)$ & 0.36 \\
Education (yr) & $12.11 \pm 2.30$ & $12.81 \pm 2.44$ & 0.06 \\
Illness duration (mo) & $40.63 \pm 22.62$ & $81.76 \pm 90.83$ & 0.18 \\
Age at onset (yr) & $25.26 \pm 7.47$ & $22.43 \pm 5.75$ & 0.67 \\
Hamilton Depression Scale & $16.53 \pm 6.22$ & $15.57 \pm 7.74$ & 0.15 \\
Positive and Negative Syndrome Scale total & $94.42 \pm 18.58$ & $101.90 \pm 13.21$ & 0.13 \\
$\quad$ Positive & $27.53 \pm 5.93$ & $30.48 \pm 6.09$ & 0.42 \\
$\quad$ Negative & $20.79 \pm 8.90$ & $22.62 \pm 4.94$ & 0.32 \\
$\quad$ General & $46.11 \pm 10.34$ & $49.29 \pm 9.48$ & 0.62 \\
Global Assessment of Functioning & $51.32 \pm 7.61$ & $50.24 \pm 6.02$ & \\
\hline
\end{tabular}

Values are presented as mean \pm standard deviation or number $(\%)$.

age, sex, years of education, or illness duration (Table 1). Table 1 displays comparisons of the baseline clinical symptom characteristics between participants in the remission and non-remission groups. The results revealed no significant differences in HAM-D, PANSS, GAF scores, or IQ between the two groups.

\section{Clinical Characteristics}

There were significant group differences in re-checked PANSS total score between the remission $(57.63 \pm 16.96)$ and non-remission $(75.90 \pm 7.96)$ groups $(p<0.001)$. Additionally, re-checked GAF scores of the remission group $(87.11 \pm 3.33)$ were also significantly different from those of the non-remission group $(63.81 \pm 8.36)(p<$ 0.001). Antipsychotic medication dose at follow-up was assessed as the mean risperidone equivalent dose [25]. Dose of remission group was $9.71 \pm 1.09 \mathrm{mg}$ and the dose of non-remission group was $10.43 \pm 1.14 \mathrm{mg}$. And the dose difference was statistically significant $(p=0.05)$. There were significant group differences in symptom changes (PANSS changes) between the remission group $(37.32 \pm 18.72)$ and non-remission $(26.95 \pm 9.96)$ groups $(p=0.03)$. The GAF changes from baseline to follow-up was also significant between the remission group $(35.79 \pm$ $7.91)$ and non-remission $(13.57 \pm 10.68)$ groups $(p<$ $0.001)$.

\section{Mismatch Negativity}

A repeated-measures ANOVA with each group as a between-subjects factor and electrode site as a within-subject factor revealed a significant main effect of the group $\left(\mathrm{F}_{1}=\right.$
Table 2. Comparison of the amplitude of the mismatch negativity between patients with schizophrenia with and without remission

\begin{tabular}{lcccc}
\hline Site $(\mu \mathrm{V})$ & $\begin{array}{c}\text { With remission } \\
(\mathrm{n}=19)\end{array}$ & $\begin{array}{c}\text { Without } \\
\text { remission }(\mathrm{n}=21)\end{array}$ & $\mathrm{t}$ & $p$ value $^{\mathrm{a}}$ \\
\hline Amplitude & & & & \\
Fz & $-2.86 \pm 1.16$ & $-1.78 \pm 1.04$ & -3.01 & $0.03^{*}$ \\
F3 & $-2.78 \pm 0.99$ & $-1.86 \pm 0.92$ & -3.04 & $0.02^{*}$ \\
F4 & $-2.93 \pm 0.98$ & $-2.22 \pm 1.10$ & -2.15 & 0.08 \\
FCz & $-2.80 \pm 1.58$ & $-1.98 \pm 1.33$ & -1.77 & 0.09 \\
FC3 & $-2.61 \pm 1.24$ & $-1.83 \pm 1.02$ & -2.18 & 0.06 \\
FC4 & $-2.82 \pm 1.10$ & $-2.17 \pm 1.30$ & -1.71 & 0.10 \\
\hline
\end{tabular}

Values are presented as mean \pm standard deviation.

${ }^{\text {a Adjusted }} p$ value using False Discovery Rate for multiple correction $\left({ }^{*} p<0.05\right)$.

7.784, $p=0.008)$. There was no significant effect on the electrode site $\left(F_{1}=1.225, p=0.29\right)$. There was no significant electrode $X$ group interaction $\left(\mathrm{F}_{5}=0.322, p=\right.$ 0.90). Independent $t$ tests of group differences in MMN peak amplitudes at each electrode showed decreased MMN amplitude in the group without remission at $\mathrm{Fz}(p=$ $0.03)$ and $\mathrm{F} 3(p=0.02)$ compared to the group with remission (Table 2). Grand-average MMN waveforms and topographical maps for each group are shown in Figure 1. The non-remitted group clearly exhibited reduced MMN amplitudes compared to those of the remitted group. MMN latencies did not significantly differ between the two groups (Table 2).

\section{Correlation Analysis of Mismatch Negativity with Clinical Symptoms and Functions}

There was a significant correlation between PANSS 


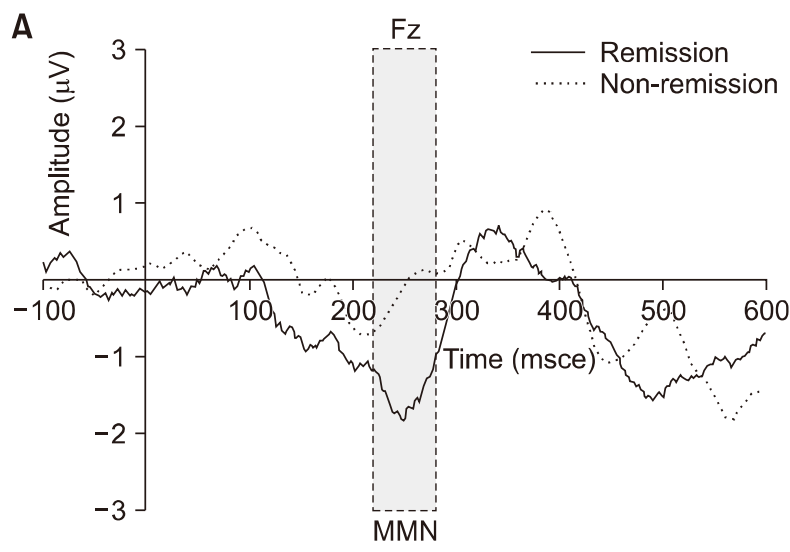

\section{B}

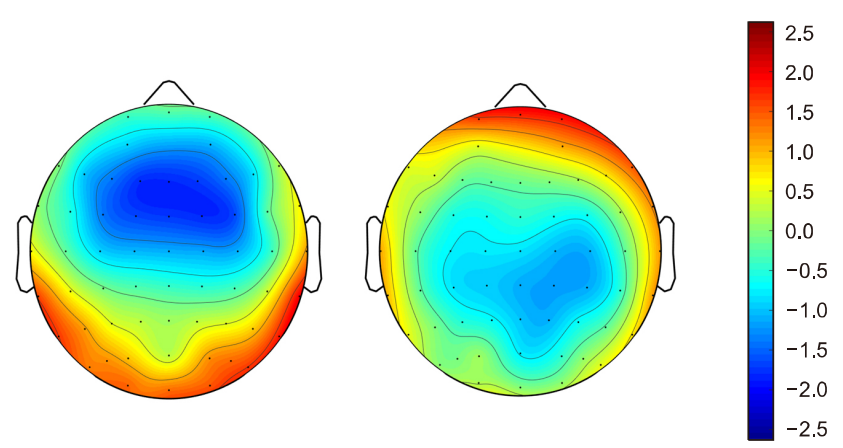

Fig. 1. (A) The grand average mismatch negativity (MMN) waveforms at $\mathrm{Fz}$ in patients with schizophrenia with and without remission. (B) Topograhic maps of $M M N$ in the schizophrenia patients with remission (left, $n=19$ ) and without remission (right, $n=21$ ).

A

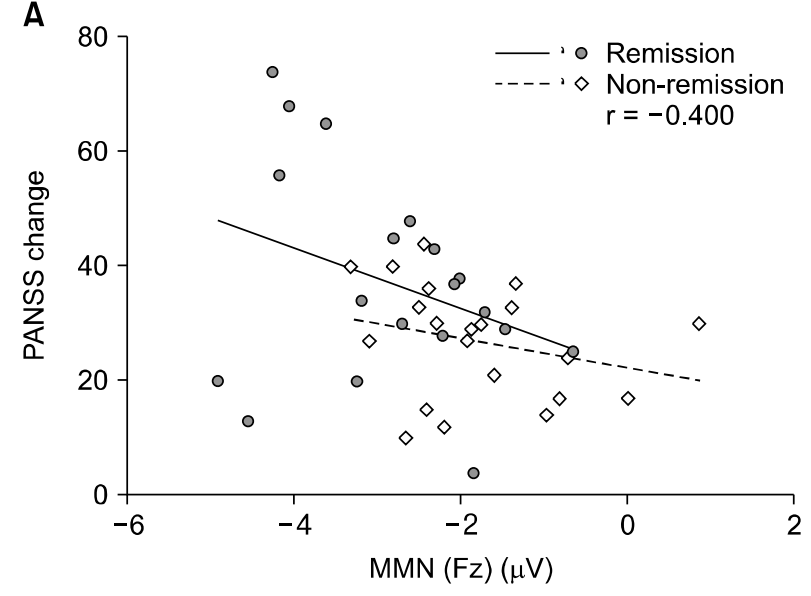

C

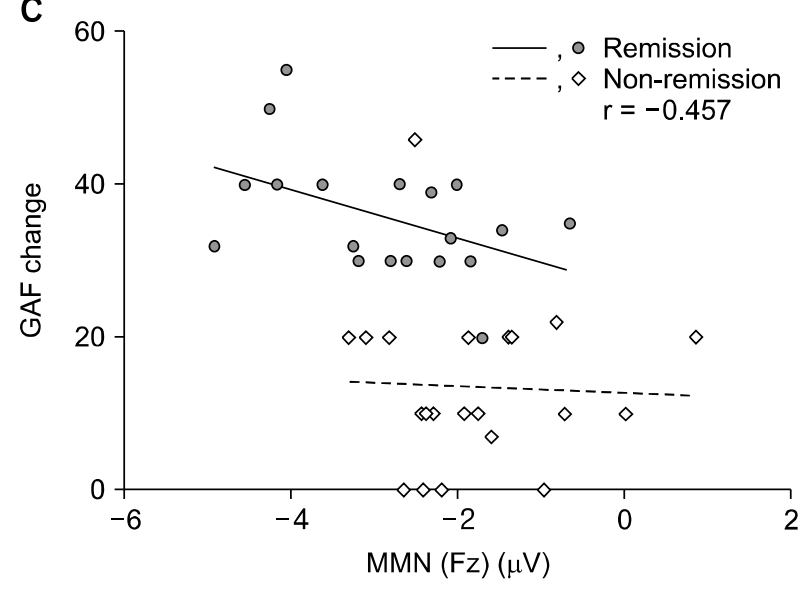

changes (differences between baseline and re-checked PANSS) and MMN peak amplitudes at $\mathrm{Fz}(\mathrm{r}=-0.400, p=$ 0.013 ) in patients with schizophrenia (Fig. 2A). A signifi cant correlation was shown between GAF changes (differences between baseline and re-checked GAF) and

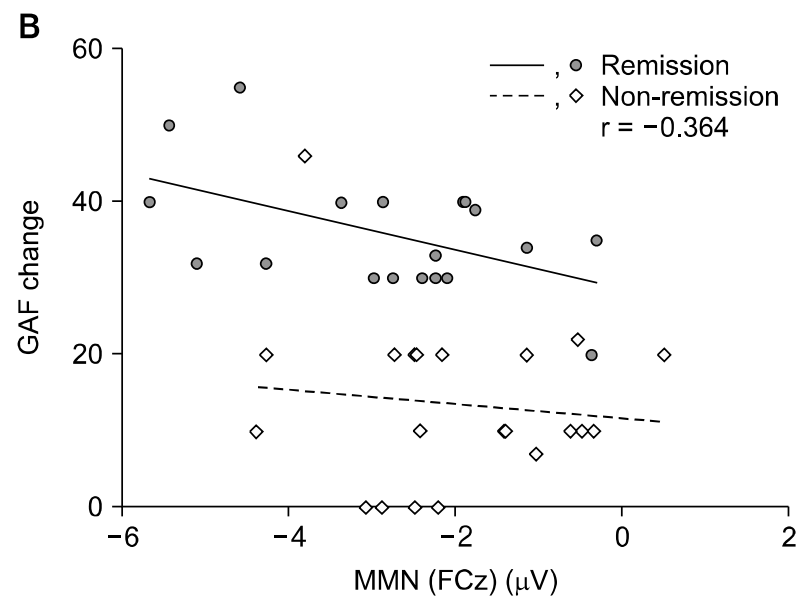

Fig. 2. (A) Scatter plots of mismatch negativity (MMN) at the Fz electrode and Positive and Negative Syndrome Scale (PANSS) changes (initially measured PANSS - re-measured PANSS). (B) Scatter plots of MMN at the FCz electrode and Global Assessment of Functioning (GAF) changes. (C) Scatter plots of MMN at the Fz electrode and GAF changes.

MMN peak amplitudes at FCz $(r=-0.364, p=0.025)$, and $\mathrm{Fz}(r=-0.457, p=0.004)$ in patients with schizophrenia (Fig. 2B, 2C). Additionally, there was a significant correlation between $\mathrm{MMN}$ peak amplitude at $\mathrm{FPz}$ and re-checked PANSS $(r=0.324, p=0.042)$, and PANSS 
Table 3. Predictors of remission and improvement of psychotic symptoms in patients with schizophrenia

\begin{tabular}{|c|c|c|c|c|}
\hline Dependent variable & Predictive variable & Standardized beta & $p$ value* & Confidence interval \\
\hline \multirow[t]{4}{*}{ Remission $^{\mathrm{a}}$} & Age & 0.504 & 0.042 & 1.019 to 2.687 \\
\hline & PANSS at baseline & 0.087 & 0.019 & 1.014 to 1.173 \\
\hline & Age at onset & -0.541 & 0.029 & 0.358 to 0.947 \\
\hline & MMN amplitude at Fz & 1.843 & 0.010 & 1.551 to 25.702 \\
\hline \multirow{4}{*}{$\begin{array}{l}\text { Improvement of psychotic } \\
\text { symptoms }\end{array}$} & PANSS at baseline & 0.546 & $<0.001$ & 0.307 to 0.846 \\
\hline & MMN amplitude at Fz & -0.336 & 0.011 & -8.343 to -1.154 \\
\hline & Antipsychotic medication dose & -0.354 & 0.008 & -8.949 to -1.483 \\
\hline & Illness duration & 0.311 & 0.041 & 0.003 to 0.147 \\
\hline
\end{tabular}

PANSS, Positive and Negative Syndrome Scale; MMN, mismatch negativity.

*The mean difference is significant at the $p=0.05$ level.

${ }^{a}$ Binary logistic regression with backward selection method.

${ }^{b}$ Multiple regression with backward selection method.

'Baseline and re-checked PANSS changes.

negative subscales $(r=0.348, p=0.028)$.

\section{Regression Analysis}

Regression analysis was conducted to predict symptom improvement and remission in patients with schizophrenia (Table 3). Multiple regression analysis was used to find predictive variables for participants' symptom improvement. The results of the regression analyses indicated that three predictors explained $53.0 \%$ of the variance " $\mathrm{R}^{2}=0.530, \mathrm{~F}(5,34)=7.673, p<0.001$ ". It was found that symptom improvement significantly predicted PANSS at baseline, illness duration, and antipsychotic dose, as did MMN amplitude at Fz. For remission, binary logistic regression tests were used. Results showed that age, age at onset, PANSS at baseline, and MMN amplitude at Fz were significant predictors of remission in patients with schizophrenia in the study. $\mathrm{R}$ square of the model was 0.513 .

\section{DISCUSSION}

This study explored the relationship of MMN with remission in patients with schizophrenia using the RSWGcr. As hypothesized, the baseline MMN at the frontal site in the remission group had larger amplitudes than those of the non-remission group. The baseline MMN amplitude, as well as some clinical characteristics, such as age, age at onset, and baseline symptom severity, predicted later remission. Moreover, the baseline MMN predicted symptom improvement in patients with schizophrenia.

The remitted patients with schizophrenia showed larger MMN amplitudes at the frontal electrode site than those of non-remitted patients. Previous studies demonstrated that early auditory information processing significantly predicted psychosocial functioning in patients with schizophrenia [28,29]. Additionally, MMN reflects functional outcomes more efficiently than do measures of neuro-cognition and theory of mind in patients with schizophrenia [30]. This study revealed that the MMN could be a significant predictor for remission and symptom improvement, as well as functional outcomes, in patients with schizophrenia. Recent studies showed that MMN was a putative predictor of symptom improvement and remission, regardless of the transition to a psychotic disorder in subjects at high clinical risk [18]. This study proposed that MMN could be associated with remission, not only in subjects at high clinical risk but also in the patients diagnosed with schizophrenia.

The present study also found that the MMN of patients with schizophrenia was significantly correlated with their PANSS total and negative symptom scores at follow-up. Several previous studies have associated decreased MMN amplitude with severity of symptoms, especially negative symptoms $[14,15,31]$. The results of this study showed that $M M N$ might be related, not only to the baseline symptom severity but also to symptom changes after treatment and functional outcomes. The study revealed that the MMN could be associated with the prognosis of schizophrenia. To clarify whether MMN amplitude predicted symptom improvement and remission, we performed regression analysis.

Our results showed that age, age at onset, initial symptom severity, and MMN amplitude at the Fz site could predict schizophrenia remission. The result was consistent 
with previous studies in which age at onset and baseline PANSS scores predicted remission $[32,33]$. Besides the clinical variables, biological markers, such as MMN, might predict schizophrenia remission. Additionally, MMN amplitude at Fz predicted improvement of psychotic symptoms. Previous studies have shown that MMN amplitude was associated with working memory deficits in patients with schizophrenia [34]. Considering the relationship between working memory and prefrontal functions, study results have suggested decreased frontal lobe functioning of schizophrenia might be related to $\mathrm{MMN}$ amplitude [35]. Given that prefrontal function might affect the acquisition of remission and symptom improvement in patients with schizophrenia, $M M N$ indices reflecting frontal functioning appeared to be a predictive factor for schizophrenia remission.

Interestingly, the final antipsychotic medication dose predicted symptom improvement, but not remission. These results were comparable to a previous study that revealed the final dose of antipsychotics could predict symptom changes, but not short-term (eight-week follow-up) remission [33]. Our study was designed to evaluate predictors for six months, a relatively long-term remission, therefore, direct comparison to Stratta and Rossi's study [33] might not be valid. Nonetheless, further evaluation of the relationship between antipsychotic dose and remission is needed.

Unexpectedly, cognition represented by the WAIS IQ was not a significant predictor for remission in this study. We previously hypothesized that IQ would affect the remission of schizophrenia because impaired neuro-cognitive function is one of the core features of schizophrenia [36], and the associations between IQ and individual tests of neuro-cognitive function have been well-studied [37]. One previous study reported that neuro-cognitive performance of patients with schizophrenia was not associated with self-reported quality of life, which was strongly associated with remission of the patients [38]. A meta-analysis demonstrated that social cognition was more strongly-associated with community functioning than neuro-cognition [39]. This suggests that the social cognition affecting remission might be different from intelligence assessed by WAIS, or that social cognition might reflect remission more than intelligence. On the other hand, Bliksted et al. [40] revealed that social cognitive deficits in schizophrenia had two distinct versions, where one was a complex, cognitive demanding form linked to IQ. The other version was related to simpler forms of social cognition and independent of IQ. Therefore, we could speculate that remission might be associated with specific social cognitive factors, independent of IQ. Our findings should be repeated with different cognitive tasks to evaluate social cognition and specific intelligence domains in remission studies with larger sample sizes.

There were a few limitations to this study. First, the participants were taking atypical antipsychotics during the study. However, a previous study revealed that the atypical antipsychotics did not appear to affect the MMN amplitude in patients with schizophrenia $[31,41]$. Moreover, this study investigated the use of antipsychotic medications and predictive values for remission and symptom improvement. Second, the relatively small sample size of this study should be considered when interpreting our results. Third, as a possible predictive factor, episode number was not considered in this study. Instead, we considered illness duration and onset age, which have been regarded to be predictors of schizophrenia remission. To avoid the effect of episode number or illness duration as covariates, we may consider evaluating patients after their first episode of psychosis. However, the diagnostic uncertainty of the population could be another consideration [42]. Future research should be conducted with specific populations (e.g., early in the course of schizophrenia), considering more possible predictive factors and covariates for remission. Last, our follow-up periods were relatively short, although previous studies used a six month follow-up period to determine attainment of remission [22]. Further studies to confirm remission after longer periods are needed. Despite the above limitations, to our knowledge, this study is the first to explore $\mathrm{MMN}$ as predictors of schizophrenia remission. Moreover, we evaluated the remission with reliable criteria, namely the RSWGcr, which objectively defined remission.

In conclusion, the $\mathrm{MMN}$ amplitude was significantly reduced in non-remitted patients with schizophrenia and correlated with symptom improvement and functional outcomes. The findings of this study suggested that MMN could be a useful biomarker for predicting symptom improvement and remission with other well-known symptomatic characteristics. 


\section{Acknowledgments}

This research was supported by the Research Foundation for Young Investigators funded by the Korean Society for Schizophrenia Research and a grant (2017R1D1A3B03030974) of the Basic Science Research Program through the National Research Foundation of Korea (NRF) funded by the Ministry of Education.

This study was also supported by Soonchunhyang University.

\section{Conflicts of Interest}

No potential conflict of interest relevant to this article was reported.

\section{Author Contributions}

Conceptualization: Ji Sun Kim, Se-hoon Shim. Data acquisition: Young Joon Kwon, Hwa Young Lee, Ho-Sung Lee. Formal analysis: Ji Sun Kim, Sungkean Kim. Writing —original draft: Ji Sun Kim. Writing—review \& editing: Ji Sun Kim, Se-hoon Shim.

\section{ORCID}

Ji Sun Kim https://orcid.org/0000-0003-2472-4591

Young Joon Kwon https://orcid.org/0000-0001-9340-0895 Hwa Young Lee https://orcid.org/0000-0002-2749-6232 Ho-Sung Lee https://orcid.org/0000-0001-6614-6465

Sungkean Kim https://orcid.org/0000-0003-4649-6112

Se- hoon Shim

\section{REFERENCES}

1. Park JH, Hong JS, Kim SM, Min KJ, Chung US, Han DH. Effects of amisulpride adjunctive therapy on working memory and brain metabolism in the frontal cortex of patients with schizophrenia: a preliminary positron emission tomography/computerized tomography investigation. Clin Psychopharmacol Neurosci 2019;17:250-260.

2. Harvey PD, Bellack AS. Toward a terminology for functional recovery in schizophrenia: is functional remission a viable concept? Schizophr Bull 2009;35:300-306.

3. Nasrallah HA, Lasser R. Improving patient outcomes in schizophrenia: achieving remission. I Psychopharmacol 2006; 20(6 Suppl):57-61.

4. Bodén R, Sundström J, Lindström E, Lindström L. Association between symptomatic remission and functional outcome in first-episode schizophrenia. Schizophr Res 2009;107:232237.

5. Brissos S, Dias VV, Balanzá-Martinez V, Carita Al, Figueira

ML. Symptomatic remission in schizophrenia patients: relationship with social functioning, quality of life, and neurocognitive performance. Schizophr Res 2011;129:133-136.

6. Valencia M, Fresán A, Barak Y, Juárez F, Escamilla R, Saracco R. Predicting functional remission in patients with schizophrenia: a cross-sectional study of symptomatic remission, psychosocial remission, functioning, and clinical outcome. Neuropsychiatr Dis Treat 2015;11:2339-2348.

7. Torgalsbøen AK, Mohn C, Rishovd Rund B. Neurocognitive predictors of remission of symptoms and social and role functioning in the early course of first-episode schizophrenia. Psychiatry Res 2014;216:1-5.

8. Kawakubo Y, Kasai K. Support for an association between mismatch negativity and social functioning in schizophrenia. Prog Neuropsychopharmacol Biol Psychiatry 2006;30:13671368.

9. Rasser PE, Schall U, Todd J, Michie PT, Ward PB, Johnston P, et al. Gray matter deficits, mismatch negativity, and outcomes in schizophrenia. Schizophr Bull 2011;37:131-140.

10. Näätänen R, Kujala T, Escera $C$, Baldeweg T, Kreegipuu $K$, Carlson $\mathrm{S}$, et al. The mismatch negativity (MMN)--a unique window to disturbed central auditory processing in ageing and different clinical conditions. Clin Neurophysiol 2012; 123:424-458

11. Näätänen R, Gaillard AW, Mäntysalo S. Early selective-attention effect on evoked potential reinterpreted. Acta Psychol (Amst) 1978:42:313-329.

12. Javitt DC, Zukin SR, Heresco-Levy U, Umbricht D. Has an angel shown the way? Etiological and therapeutic implications of the PCP/NMDA model of schizophrenia. Schizophr Bull 2012;38:958-966.

13. Light GA, Braff DL. Mismatch negativity deficits are associated with poor functioning in schizophrenia patients. Arch Gen Psychiatry 2005;62:127-136.

14. Salisbury DF, Shenton ME, Griggs CB, Bonner-Jackson A, McCarley RW. Mismatch negativity in chronic schizophrenia and first-episode schizophrenia. Arch Gen Psychiatry 2002; 59:686-694.

15. Umbricht DS, Bates JA, Lieberman JA, Kane JM, Javitt DC Electrophysiological indices of automatic and controlled auditory information processing in first-episode, recent-onset and chronic schizophrenia. Biol Psychiatry 2006;59:762772.

16. Kim M, Kim SN, Lee S, Byun MS, Shin KS, Park HY, et al. Impaired mismatch negativity is associated with current functional status rather than genetic vulnerability to schizophrenia. Psychiatry Res 2014;222:100-106.

17. Umbricht D, Krljes S. Mismatch negativity in schizophrenia: a meta-analysis. Schizophr Res 2005;76:1-23.

18. Kim M, Lee TH, Yoon YB, Lee TY, Kwon JS. Predicting remission in subjects at clinical high risk for psychosis using mismatch negativity. Schizophr Bull 2018;44:575-583.

19. Andreasen NC, Carpenter WT Jr, Kane JM, Lasser RA, Marder 
SR, Weinberger DR. Remission in schizophrenia: proposed criteria and rationale for consensus. Am I Psychiatry 2005; 162:441-449.

20. Lambert M, Karow A, Leucht S, Schimmelmann BG, Naber D. Remission in schizophrenia: validity, frequency, predictors, and patients' perspective 5 years later. Dialogues Clin Neurosci 2010;12:393-407.

21. van Os J, Burns T, Cavallaro R, Leucht S, Peuskens J, Helldin $\mathrm{L}$, et al. Standardized remission criteria in schizophrenia. Acta Psychiatr Scand 2006;113:91-95.

22. Fukumoto M, Hashimoto R, Ohi K, Yasuda Y, Yamamori H, Umeda-Yano S, et al. Relation between remission status and attention in patients with schizophrenia. Psychiatry Clin Neurosci 2014;68:234-241.

23. First MB, Gibbon M, Spitzer RL, Williams JBW, Benjamin LS. User's guide for the structured clinical interview for DSM-IV axis II personality disorders: SCID-II. Washington, DC:American Psychiatric Press; 1997.

24. Burkey JM, Lippy WH, Schuring AG, Rizer FM. Clinical utility of the 512-Hz Rinne tuning fork test. Am J Otol 1998;19: 59-62.

25. Leucht S, Samara M, Heres S, Patel MX, Woods SW, Davis JM. Dose equivalents for second-generation antipsychotics: the minimum effective dose method. Schizophr Bull 2014;40: 314-326.

26. Pinna F, Tusconi M, Bosia M, Cavallaro R, Carpiniello B; Cagliari Recovery Group Study. Criteria for symptom remission revisited: a study of patients affected by schizophrenia and schizoaffective disorders. BMC Psychiatry 2013; 13:235.

27. Semlitsch HV, Anderer P, Schuster P, Presslich O. A solution for reliable and valid reduction of ocular artifacts, applied to the P300 ERP. PSychophysiology 1986;23:695-703.

28. Thomas ML, Green MF, Hellemann G, Sugar CA, Tarasenko $\mathrm{M}$, Calkins ME, et al. Modeling deficits from early auditory information processing to psychosocial functioning in schizophrenia. JAMA Psychiatry 2017;74:37-46.

29. Wynn JK, Sugar C, Horan WP, Kern R, Green MF. Mismatch negativity, social cognition, and functioning in schizophrenia patients. Biol Psychiatry 2010;67:940-947.

30. Lee SH, Sung K, Lee KS, Moon E, Kim CG. Mismatch negativity is a stronger indicator of functional outcomes than neurocognition or theory of mind in patients with schizophrenia. Prog Neuropsychopharmacol Biol Psychiatry 2014;48:213-
219.

31. Catts SV, Shelley AM, Ward PB, Liebert B, McConaghy N, Andrews $\mathrm{S}$, et al. Brain potential evidence for an auditory sensory memory deficit in schizophrenia. Am J Psychiatry 1995; 152:213-219.

32. Immonen J, Jääskeläinen $\mathrm{E}$, Korpela $\mathrm{H}$, Miettunen J. Age at onset and the outcomes of schizophrenia: a systematic review and meta-analysis. Early Interv Psychiatry 2017;11:453-460.

33. Stratta P, Rossi A. Short-term remission in schizophrenia as a combination of several outcome measures. Psychiatry Res 2013;209:401-405.

34. Javitt DC, Doneshka P, Grochowski S, Ritter W. Impaired mismatch negativity generation reflects widespread dysfunction of working memory in schizophrenia. Arch Gen Psychiatry 1995; 52:550-558.

35. Harms MP, Wang L, Csernansky JG, Barch DM. Structurefunction relationship of working memory activity with hippocampal and prefrontal cortex volumes. Brain Struct Funct 2013;218:173-186.

36. Green MF, Harvey PD. Cognition in schizophrenia: past, present, and future. Schizophr Res Cogn 2014;1:e1-e9.

37. Mohn C, Sundet K, Rund BR. The relationship between IQ and performance on the MATRICS consensus cognitive battery. Schizophr Res Cogn 2014; 1:96-100.

38. Haro JM, Novick D, Perrin E, Bertsch J, Knapp M. Symptomatic remission and patient quality of life in an observational study of schizophrenia: is there a relationship? Psychiatry Res 2014; 220:163-169.

39. Fett AK, Viechtbauer W, Dominguez MD, Penn DL, van Os J, Krabbendam $\mathrm{L}$. The relationship between neurocognition and social cognition with functional outcomes in schizophrenia: a meta-analysis. Neurosci Biobehav Rev 2011;35:573-588.

40. Bliksted V, Fagerlund B, Weed E, Frith C, Videbech P. Social cognition and neurocognitive deficits in first-episode schizophrenia. Schizophr Res 2014;153:9-17.

41. Umbricht D, Javitt D, Novak G, Bates J, Pollack S, Lieberman $\mathrm{J}$, et al. Effects of risperidone on auditory event-related potentials in schizophrenia. Int J Neuropsychopharmacol 1999;2: 299-304.

42. Kim JS, Baek JH, Choi JS, Lee D, Kwon JS, Hong KS. Diagnostic stability of first-episode psychosis and predictors of diagnostic shift from non-affective psychosis to bipolar disorder: a retrospective evaluation after recurrence. Psychiatry Res 2011; 188:29-33. 